\title{
Research on the Novel E-Commerce Recommendation Framework based on Modified NN Model and the Collaborative Analysis Paradigm
}

\author{
Ning Wang ${ }^{1,2,3}$, Mingming Chen ${ }^{1,3,6 *}$, Qiaoling Zhang ${ }^{4}$ and Liejun Yang ${ }^{5}$ \\ ${ }^{l}$ Dept. of Information and Mechatronics Engineering, Xiamen Huaxia University, \\ Xiamen, Fujian, China \\ ${ }^{2}$ Dept. of Automation, Xiamen University, Xiamen, Fujian, China \\ ${ }^{3}$ Fujian Province Engineering Research Center on New Generation of Information \\ and Communication Technology and Wisdom Education, China \\ ${ }^{4}$ Dept. of Commerce, Xiamen Huaxia University, Xiamen, Fujian, China \\ ${ }^{5}$ Dept. of Computer Science, Ningde Teachers' College, China \\ ${ }^{6}$ University of Illinois at Springfield, United States, USA \\ *Mingming Chen
}

\begin{abstract}
In this paper, we conduct research on novel E-commerce recommendation framework based on modified NN model and collaborative analysis. This article trusting relationship as an important attribute of influence recommendation, will use the condition transitivity of trusting relationship, designs and constructs a blending trust network, and selects the two-dimensional similar close neighbors of interest-based and trust dual factor for the target users based on this. We proposed the method based on two essential tools, (1) while classical parametric techniques are helpless to the modeling of many natural and social phenomena, neural network techniques provide shortcuts. The function of the artificial neural network and information processing capacity is determined by structure; (2) The combination of the two algorithms can make use of the advantages of the algorithm based on content, similarity matching of the project, especially when a project that has not been without the user's evaluation that can also recommend to the user. We numerically analyze the integration model of the NN and the RS. The experiment result proves the effectiveness of the method. From the experimental simulation curve, we could conclude that the proposed method achieves better accuracy.
\end{abstract}

Keywords: E-commerce; Recommendation Framework; Neural Network; Collaborative Analysis; Paradigm; Systematic Design

\section{Introduction}

With the rapid development of the Internet, online shopping becomes one of the most important ways of the shopping consumer. In the face of the shopping website product information, the amount of the recommendation system to help users quickly searches for products that meet the requirements. In the numerous recommendation technologies, the application of the collaborative filtering recommendation technology is more extensive. Under normal conditions, recommends the style of the computation and recommendation explanation of result is uninterested. The main purpose that recommendation explained was to improve user's confidence to the recommendation system, for this reason usually needs to promote recommendation system the transparency. Allow the user to understand

${ }^{*}$ Corresponding Author 
that the recommendation system the operational mechanism can enhance the user's trust degree to the recommendation system [1-3].

Different from the traditional information filtering technology of the search engines, recommendation systems do not need to provide for search keywords, it will through the analysis of that the historical transaction records of the user or the user behavior mining potential interest, and to recommend, therefore, the recommendation system to meet the personalized requirements of users [4-6]. The following will introduce the recommended one by one interpretation. We analyze in detail in the table one.

Table 1. The Recommended One by One Interpretation

\begin{tabular}{|c|c|}
\hline Recommended Interpretation Type & Explain \\
\hline $\begin{array}{c}\text { Case-based recommendation } \\
\text { explanation }\end{array}$ & $\begin{array}{l}\text { The recommendation explanation of CBR } \\
\text { regarding the classics solution of this issue } \\
\text { is, the demand of user with giving the } \\
\text { example compares, and stressed that } \\
\text { which restrictive conditions have been } \\
\text { satisfied, which do not have. }\end{array}$ \\
\hline $\begin{array}{l}\text { Recommendation based on } \\
\text { knowledge and natural language }\end{array}$ & $\begin{array}{l}\text { A recommendation system based on } \\
\text { knowledge and natural language will give } \\
\text { priority to the use of the content } \\
\text { information of the item, that is, the } \\
\text { attribute, as an explanation of its } \\
\text { recommendation. }\end{array}$ \\
\hline $\begin{array}{l}\text { Population statistics-based } \\
\text { recommendation explanation }\end{array}$ & $\begin{array}{l}\text { Demographic-based recommendation } \\
\text { interpretation takes user A's demographic } \\
\text { information as input, and according to this } \\
\text { information, the recommendation } \\
\text { algorithm identifies a set of users that are } \\
\text { similar to A in the population distribution. }\end{array}$ \\
\hline Content based recommendation & $\begin{array}{l}\text { The prerequisite scenario for this } \\
\text { interpretation is that the user is browsing a } \\
\text { particular item of interest and analyzing } \\
\text { other products of interest to the user by } \\
\text { finding a set of users similar to the user. }\end{array}$ \\
\hline $\begin{array}{l}\text { Collaborative based } \\
\text { recommendation interpretation }\end{array}$ & $\begin{array}{l}\text { It does not use user's grading information } \\
\text { to the goods, but calculates the similarity } \\
\text { between goods using the contents attribute } \\
\text { of the goods. }\end{array}$ \\
\hline
\end{tabular}

According to the theoretical review, the corresponding methodologies of the current recommendation algorithms can be summarized as the listed aspects.

- Based on collaborative filtering (CF) recommendation technology. Collaborative filtering algorithm generally includes the collaborative filtering recommendation algorithm based on memory and the collaborative filtering algorithm based on the project [7-8].

- Recommendation technology based on association rules. The technology is using association rules and find out the correlation between the transactions. If the rules of each interest group as former, then rules after the piece is the recommendation of the interest group and must be the target [9].

- Based on the recommendations from the basic content filtering (BCF) technology. Recommended based on content filter is the similarity between user interest and 
system resources calculation, while the relevance of some of the larger resources recommended to the user [10-12].

This article will trust in the introduction collaborative filtering (CF) recommendation algorithm, thinks that the trust is in the system some user after the synthesis understood other user history behaviors, recommends the one approval of behavior to them that is to the one subjective affirmation of authenticity, secure and use value these recommendation information which will change with the time and other factor changes. Recommended in this paper, the existing evaluation index system for the review of the system, and the recommendation system evaluation index of latest research progress were summarized, from the accuracy, variety, novelty, and shed light on the multiple perspectives such as coverage. In the later sub-section, we will systematically build up the framework of the method and will conduct experimental analysis. To start, in the figure one, we show the classification of the current existing RS methodologies

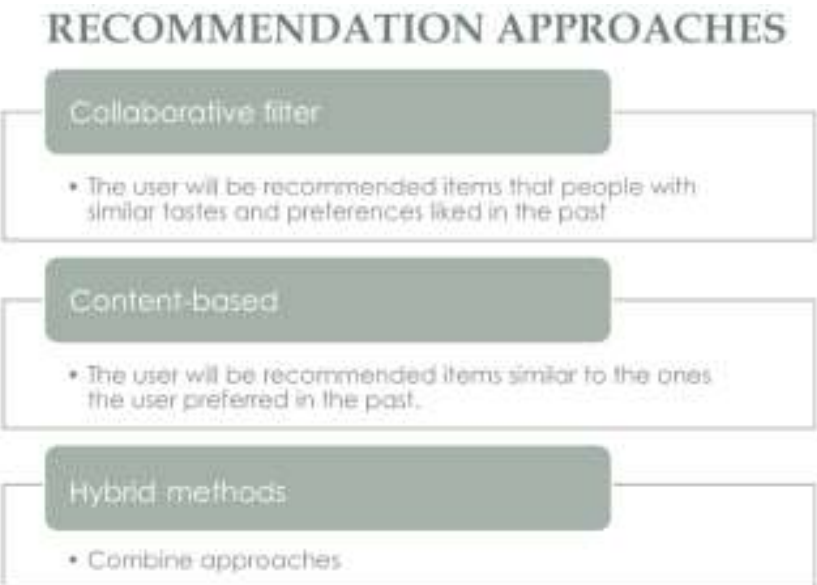

Figure 1. The Classification of the Current RS Methodologies

\section{The Neural Network and the Modification Paradigm}

Neural network belongs to the intelligent machine, which can obtain the effective problem-solving characteristic through the study, thus attracts many researchers and the application. While classical parametric techniques are helpless to the modeling of many natural and social phenomena, neural network techniques provide shortcuts. The function of the artificial neural network and information processing capacity is determined by its structure. At present, the application of the artificial neural network mostly adopts the forward multi-layer network and B P algorithm which needs major modification [13-14].

Associative memory is one of the important applications of artificial neural network. It is through training sample set and makes sample network steady state as the associative memory is stored on the stable attractor [15]. When those with incomplete or change the information of data, will enable the network to call stored sample pattern that will make information is populated or correction which gives the correct result, this is the tolerance of memory neural network. In the formula one, we show the architecture.

$$
\begin{aligned}
& x(t+1)=f \sum_{i=1}^{M} V_{i j} \sum_{i=0}^{i} k^{\prime} A_{i}(t-d) \\
& +\sum_{F=1}^{N} W_{0} \sum_{i=0}^{1} k_{0} x_{i}(t-d) \\
& \left.-T \sum_{t=0}^{t} k_{t} g(x)(t-d)\right)-\theta_{i} 1
\end{aligned}
$$


The feedforward neural network achieves structural optimization by minimizing the number of connection weights. Compared with other methods, this optimization method has several advantages: the network structure is simpler, the learning speed is faster and the induction performance is better. The structure optimization of the feedforward neural network includes the following points.

- The number of connection weights between neurons was determined.

- Rule, by the model to find the optimal structure, mainly through the training and comparison of different network structure of the way to achieve that will highly respected method of cross validation.

- Bottom-up the method, starts from the relatively small structure, then gradually increases the concealed neuron quantity, while then thus strengthens the network performance.

When the neural network input with fault tolerance of the neural network can attract domain into chaos, chaos movement after a certain dynamic movement, can always reach the chaotic attractor, and finally to achieve correct discriminant fault categories. When the neural network input fault tolerance beyond a certain range, the network of the chaotic movement from the original attraction domain, and the final convergence is less than the original chaotic attractor as follows [15-16].

$$
\begin{aligned}
y(t+1) & =\left[k y r(t) \sum_{j=1}^{n} W_{i j x}(t)-\mathrm{T}_{x+a}+a\right. \\
& \left.+\sum_{i=0}^{N=0} W_{i}-t k-1\right) g^{i}(t) \\
g^{t}(t)= & B K^{*}\left(x_{i}(t)-\tilde{x}^{i}\right)
\end{aligned}
$$

The most critical problem of neural network is the establishment of non-linear mapping between input and output, and that the stronger nonlinear mapping ability, with the better performance of network structure. From the systematic angle, the BP can be separated into the two stages [17-18]. (1) Forward communication. The input samples from the input layer, through the hidden layer, layer by layer processing, and finally output from the output layer. (2) Back propagation. If the output is not equal to the expectation, reverse propagation occurs. Starting from the output layer, the error signal in the original way of positive transmission back to the original, and the hidden layer of the neurons of the weight coefficient correction, the deviation of the signal tends to minimize.

For the further optimization of the issues, we should focus on the structure and essence part kernel modification [19-21]. The design of the fuzzy rule uses to collect the method based on the experience for focus trying, to guarantee the network precision that is often partial in the choice many enough fuzzy rule numbers, will inevitably create the network the redundancy, increased the computation load, the regular quantity of network becomes along with the input variable integer the exponent relation increases generally [22]. In the formula 4 , we define the architecture of the fuzzy neural network.

$$
u_{f u z z y}=\frac{\sum_{k} W_{k}\left(\prod_{i=1}^{n} \mu_{F i j}\left(x_{i}\right)\right)}{\sum_{k}\left(\prod_{i=1}^{n} \mu_{F i j}\left(x_{i}\right)\right)}
$$

The structure and parameters of the fuzzy neural network are the parameters including the fuzzy membership function, the number of fuzzy rules and the weighting parameters. The key to the optimization of the fuzzy network structure is how to find the essential 
compact fuzzy rule set, therefore, in its accuracy and complexity of the network structure between the balance, to find the optimal network, for which a performance index.

$J_{\text {objective }}=\min \left(\log _{e}(E)+\lambda \frac{n_{\text {regulation }}}{\max L}\right)$

Correctly chooses the initial network and optimization studies and improves induction be closely linked. Regarding the initial network, we can have the innumerable choices, but only has little suits the actual problem with gives the issue the complexity to compare, the initial network that if chooses is too small, then induction of obtained network will be very bad; However, the initial network that if chooses is oversized that will waste the resources and time. Once can select suitable initial network, it will then urge the obtained network to be simpler, the learning speed to be quicker and induction is better.

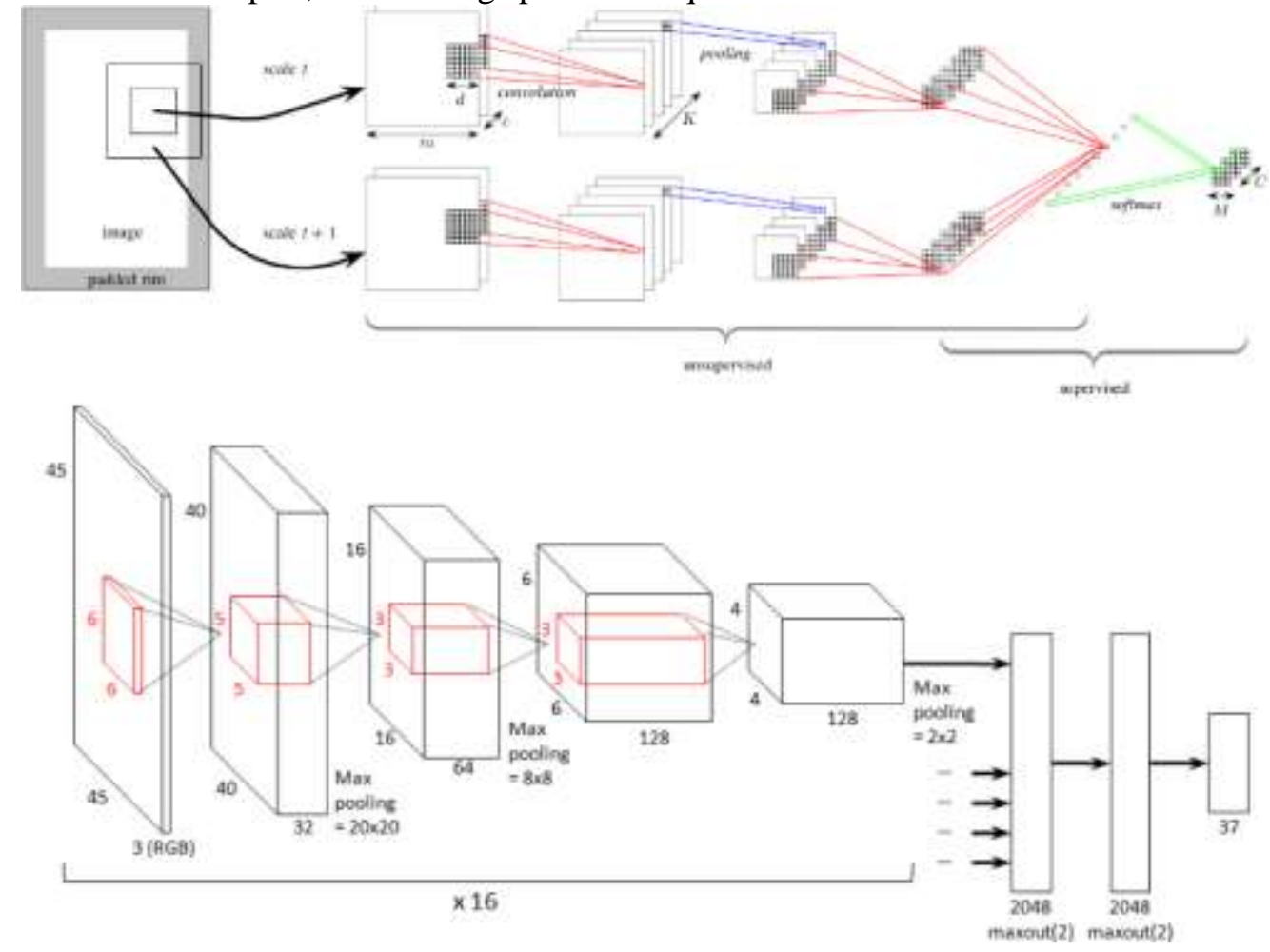

Figure 2. The Revised Neural Network Structure

The structural complexity of the canonical fuzzy neural network mainly depends on the number of fuzzy rules, the number of redundant fuzzy rules makes the network redundant, and a considerable number of them are invalid rules. Network the membership function parameter and network architecture of (i.e., fuzzy rule number) after regarding chaos optimization maintains the invariable, network equal in series expansion of a series of the primary function. The core adjustable parameter in the primary function expansion of this network is linear, therefore, may use OLS online adjustment power parameter $\mathrm{W}$ realizes the adaptive control of network. Therefore, we should consider adjusting the parameters of the neural network based on the formula 6 .

$\Delta W=-\frac{W_{i}}{\left[H^{-1}\right]} H^{-1}$

While, we should also consider the listed parameters. 


$$
\begin{aligned}
& Y=\left[\begin{array}{c}
u[x(1)] \\
u[x(2)] \\
u[x(3)] \\
\vdots \\
u[x(N)]
\end{array}\right] . \\
& Q=\left[\begin{array}{cccc}
Q_{11} & Q_{12} & \cdots & Q_{\mathrm{v}} \\
Q_{21} & Q_{22} & \cdots & Q_{\mathrm{vV}} \\
\vdots & & & \vdots \\
Q_{\mathrm{N}} & Q_{\mathrm{r}} & \cdots & Q_{\mathrm{rr}}
\end{array}\right], \\
& W=\left[\begin{array}{c}
w_{1} \\
w_{2} \\
w_{3} \\
\vdots \\
w_{T}
\end{array}\right], E=\left[\begin{array}{c}
e_{1} \\
e_{2} \\
e_{3} \\
\vdots \\
e_{T}
\end{array}\right]
\end{aligned}
$$

According to the principle of pruning technology and the idea of that following OBS algorithm, the improved algorithm is based on the second derivative of error function, from the basic idea of the balance between network complexity and training error, and then optimizes the network structure [23]. The revised architecture can be show as below.

$$
\Delta W_{i j} \alpha=\frac{\partial e}{\partial W_{i j}}
$$

\section{The Proposed Methodology}

\subsection{Collaborative Analysis and Electronic Commerce}

The cooperative awareness is also one of CSCW research hot topics. In joint operation process, sensation information for efficiency of enhancement joint operation necessary cooperation relevant information. The sensation information should be divided into the static sensation and dynamic sensation information, humanity is quite often sensitive to the sensation of the dynamic sensation information [24-25]. In order to make the whole system operate effectively, correctly and carefully classify different levels of perception is an important task in the realization of collaborative e-commerce system. The perceptual information may contain the following contents.

- Time: This some described this user whether as well as when once was getting online in online.

- In the field of work in different circumstances, for the same level of importance of information will inevitably be different, for example, the user in advertising design for the new drawing technical information will be more attention than the other users.

- Focus: Sometimes users perceive different requirements, such as that a user at the same time opened a number of windows to work, when he was dealing with other things may not want to be too much interference and want to know only certain specific information.

The working space sensation already can regard as one result, can regard as a process. The so-called result is one type about other coordination through the working space interactive understanding condition, here working space allows the participant to explain the event, forecast that the demand and carries on the suitable interaction. The so-called process is one type extracts the information from the coorperate environment continually 
the cyclic process, according to existing knowledge integration the information, and will use these knowledge instruction future sensations [26-29].

In order to achieve dynamic co-sense, the application server and each client resides in a co-sense agent. Wherein the co-aware agent in the application server also maintains a perceptual table and a Notification server and the perceptual table holds information about the current status of all online users, such as user names, location, actions, concerns, etc. For this term, the Agent based method will be more efficient and effective [30]. The users visit each terminal that the electronic commerce system uses with the terminal Agent connection, terminal Agent monitor the user behavior, and establishes the user with the local configuration files that this terminal is connected; Each user is connected user Agent, the user Agent collection terminal Agent information that establishes the user by chance configuration files, and divides according to the configuration files by chance. The Agent parameters can be defined as formula 9 .

Measured $_{a b}=\frac{\left|G U C F_{a} \cap G U C F_{b}\right|}{\left|G U C F_{a} \cup G U C F_{b}\right|}$

In short, the user Agent to calculate the similarity of the user's decision and similarity is dynamically recalculated and partially overlapped (a user can belong to a different partition). This indicates clustering is performed in offline mode, completely independent of user behavior. Therefore, clustering does not affect the efficiency of the user behavior, especially in the recommended generation process. However, this clustering architecture is not optimal because clustering is performed cyclically, rather than when data changes.

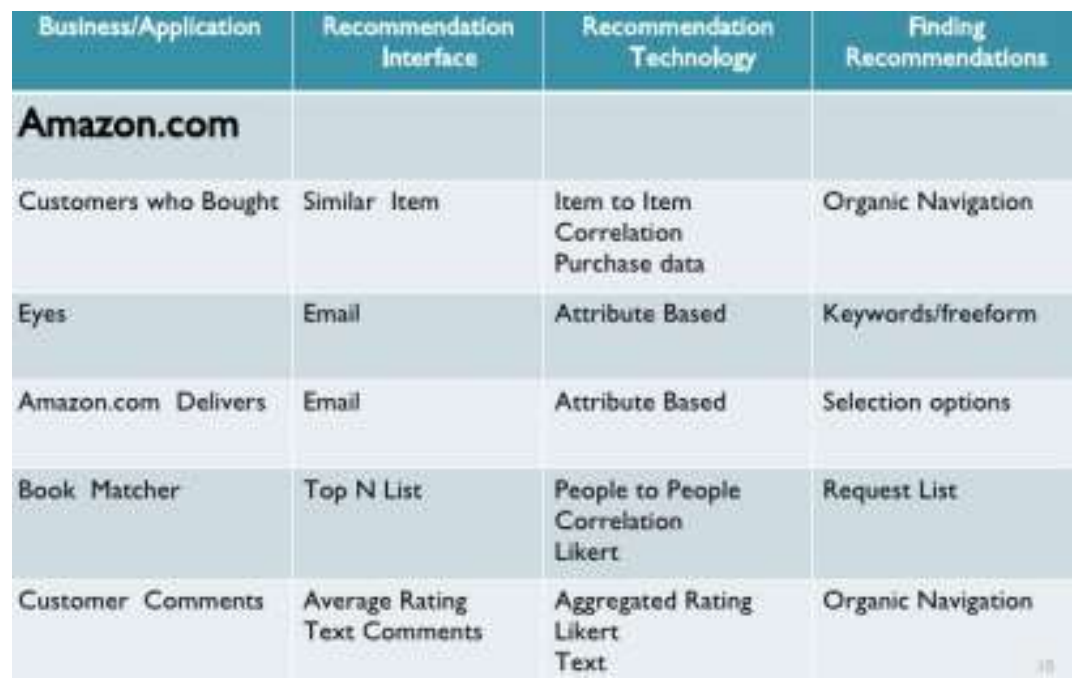

Figure 3. The Sample Illustration of the Collaborative Analysis Pattern

\subsection{The Proposed Paradigm}

In order to establish a reasonable user model to meet different users, to the requirement of real-time and recommend ways to produce a series of recommended techniques and the algorithms, we should consider the listed issues [31-36].

- The combination of the collaborative filtering and content-based algorithm. The combination of the two algorithms can make use of the advantages of the algorithm based on content, similarity matching of the project, especially when a project that has not been without the user's evaluation that can also recommend to the user.

- Collaborative filtering and combination algorithm based on association rules. Technology for collaborative filtering system is the use of basic association rules Apriori algorithm through mining the user's evaluation records related to that recommend. 
- $\quad$ Based on the model of the algorithm only scans the database user ratings again can finish is recommended for all users. Advantage is to establish the model of much smaller compared to the original data set, so that can effectively alleviate the real-time problem of recommendation algorithm.

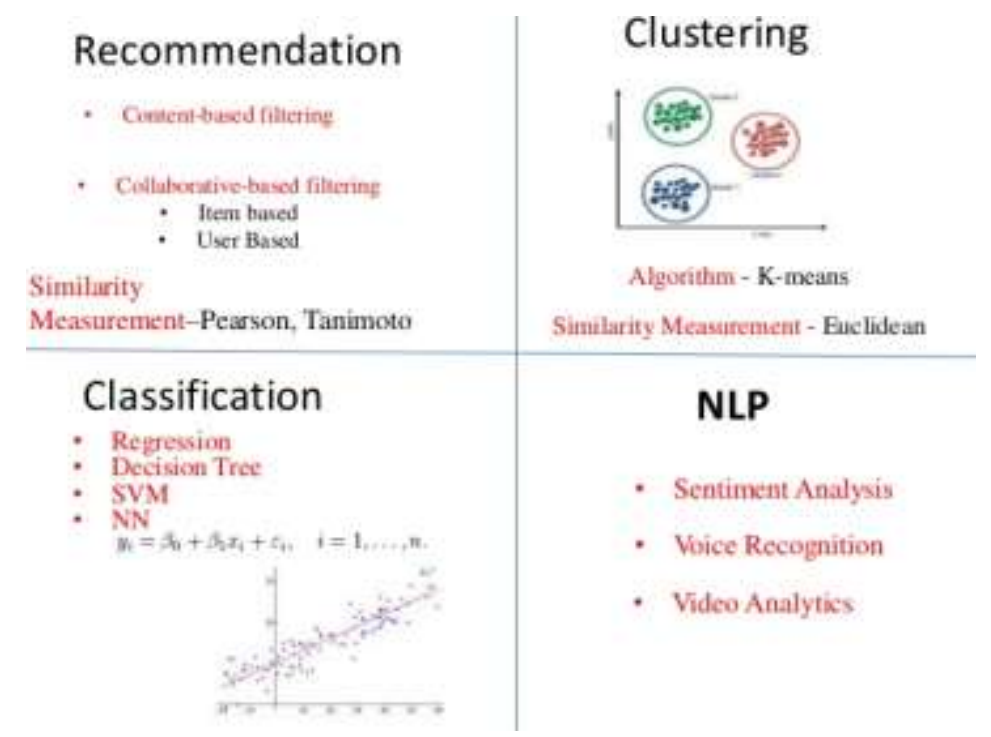

\section{Figure 4. The Comparison Analysis of the Different Algorithms}

In the collaborative filtering algorithm, while it is undoubtedly the key of the whole algorithm to determine the nearest neighbor of the target user, however, the target user nearest neighbor is determined by calculating the similarity between the target user and the other user. Therefore, the calculation of the similarity between users is the key to the accuracy of the entire system. To achieve this, we should consider the following issues.

- In carries on the initialization after the trust network that uses the partial trust network of the corresponding trusting relationship transmission rule in user to the network to expand, gains among the users the indirect trusting relationship.

- If there is trust relationship among the users, there is a directed edge between the corresponding nodes in the graph. The weight of the edge represents the trust value between the users.

Most recommendation algorithms base on the static data, has not considered the growth of user and goods, after the new user and goods join, as the recommendation system needs to carry on to the new tensor decomposes, this, had decomposed the tensor data must re-computat has wasted the large amounts of resources. To solve this problem, we proposed tensor decomposition algorithm that the dynamic gain renews. This method does not need to re-computate to primitive tensor that is new tensor of result and new user and goods constitution that uses the primitive tensor to decompose that carries on the dynamic updating to primitive decomposition result. For this, we define the similarity means as the following formula 10.

$$
\text { Similarity }_{\text {measure }}=\cos (I, J)
$$

In the real environment, the similarity between the two users is not only related to the score of the project evaluation, but also related to the user's own interest in a project. When two users have the same interests as it is easy to think that they have a high degree of similarity. In the Figure 5, we show the feature. 


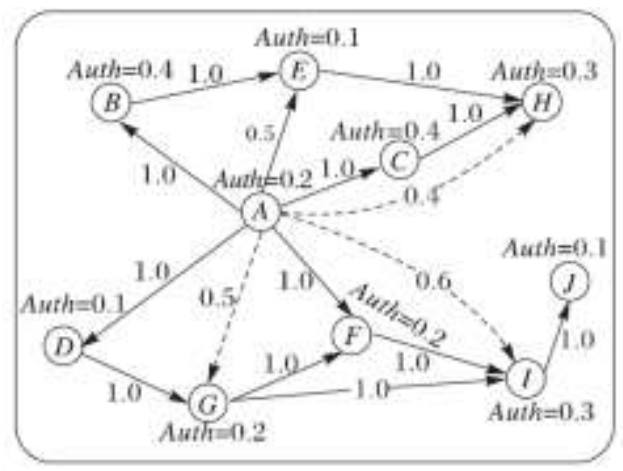

\section{Figure 5. The Similarity Modelling Procedure}

In the cosine similarity measurement method, the problem of different users' evaluation scale is neglected. In order to improve the problem of the cosine similarity measurement method, the modified cosine similarity measure subtracts the average score of the user. The revised element will be revised as formula 11 .

$$
\text { Similarity }_{\text {measure }}=\cos (I, J)+\Gamma
$$

The similarity between users is influenced and restricted by the factors of interest and trust. In order to reflect the common effect of the two factors in the selection of similar neighbors in the recommendation process, the similarity value and the trust evaluation value need to be evaluated before the score prediction, reconciliation as the use of the statistics in the commonly used weighted harmonic mean calculation method.

\section{Experiment and Verification}

In this paper, we conduct the simulation. This article uses the real data set to carry on the validating experiment to the algorithm that this article proposed. It is a service online website, the user can grade the goods on the website and can join somebody to own trust list in. The accuracy of recommendation is an important measure to evaluate the quality of recommendation system, which depicts the matching degree between the user's predictive value and the actual value. The following results reflect the effectiveness of the method.

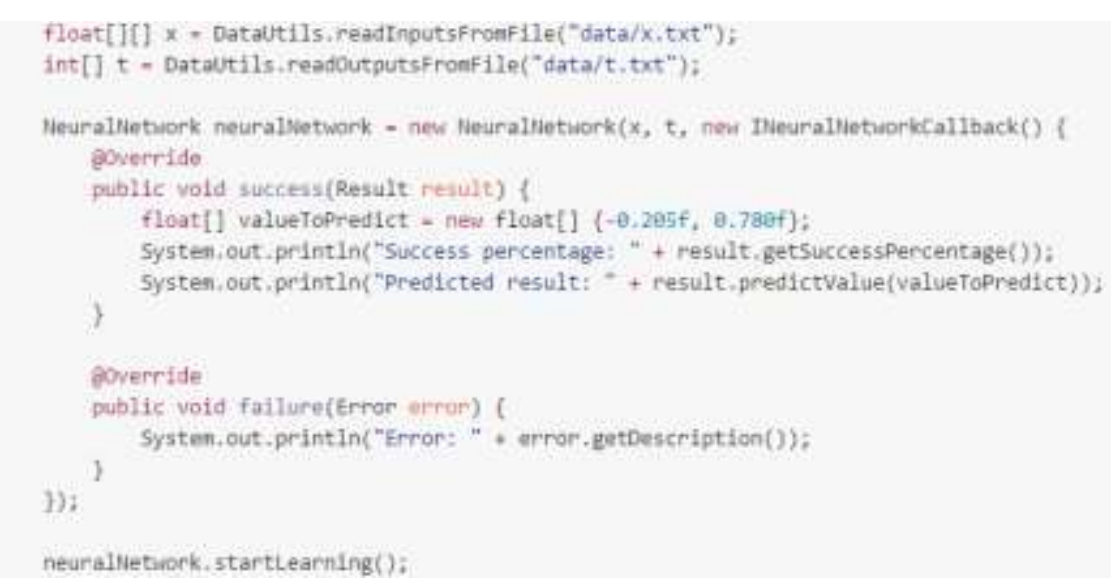

Figure 6. The Configure Code for the System 

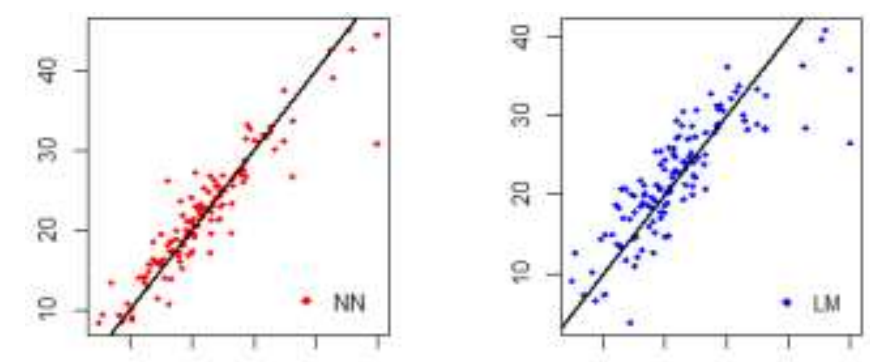

$\begin{array}{lllll}10 & 20 & 30 & 40 & 50\end{array}$

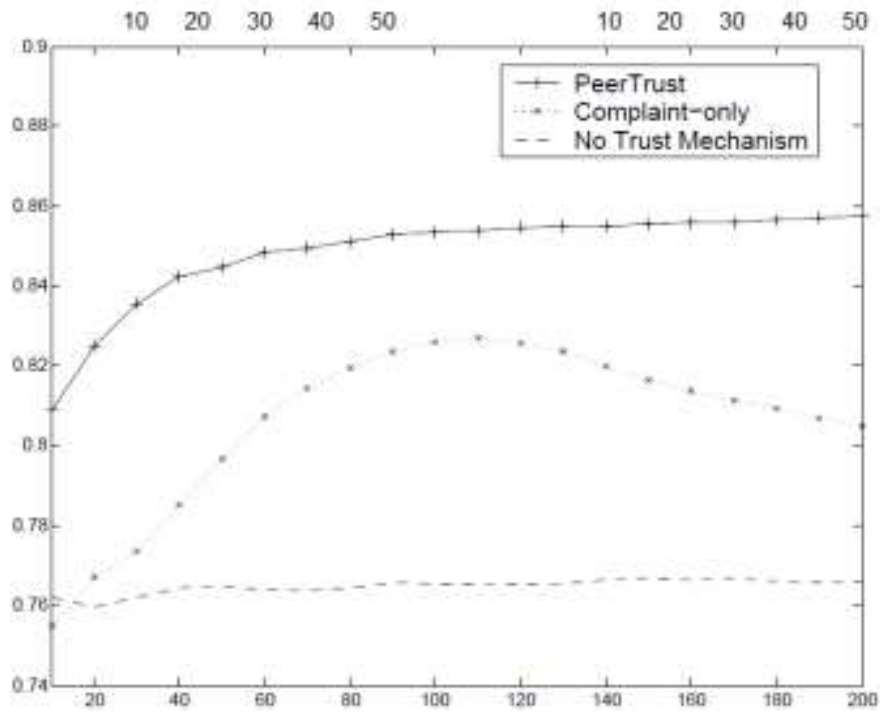

Figure 7. The Numerical Simulation Result

\section{Conclusion}

In this paper, we conduct research on novel E-commerce recommendation framework based on modified NN model and the collaborative analysis. This paper analyzes the shortcomings of the traditional similarity measure method in the case of user scoring sparseness. In view of these shortcomings, the user interest degree is introduced into the calculation of user similarity, and a similarity measure based on the user interest degree is proposed, the algorithm at each incremental step to the original data and the increment data decomposition and the new data set to do arithmetic, so time will have larger growth algorithm. It can improve the scalability of the system, and make the system more adaptable to the change of the commodity and the user, and then improve the reliability of the system. The effectiveness of the method is proved, in the near future, we will conduct more research to enhance the performance the current model.

\section{Acknowledgments}

This paper is financially supported by the Education and Research Project of Fujian Province (NO. JA15877); Science and Technology Project of Xiamen (NO. 3502Z20163015).

This paper is a revised and expanded version of a paper entitled [A Novel E-commerce Recommendation Algorithm based on Neural Network and Collaborative Analysis] presented at [ISI 2016]. 


\section{References}

[1] J. Wang and Y. Zhang, "Opportunity model for e-commerce recommendation: right product; right time", In Proceedings of the 36th international ACM SIGIR conference on Research and development in information retrieval. ACM, (2013), pp. 303-312.

[2] Y. M. Li, C. T. Wu and C. Y. Lai, "A social recommender mechanism for e-commerce: Combining similarity, trust, and relationship", Decision Support Systems, vol. 55, no. 3, (2013), pp. 740-752.

[3] Z. Gao, Z. Li and K. Niu, "Solutions for problems of existing E-Commerce recommendation system." In Consumer Electronics-Taiwan (ICCE-TW), 2016 IEEE International Conference on. IEEE, (2016), pp. $1-2$.

[4] Y. S. Cho, S. C. Moon and K. H. Ryu, "Mining association rules using RFM scoring method for personalized u-commerce recommendation system in emerging data", In Computer Applications for Modeling, Simulation, and Automobile, pp. 190-198. Springer Berlin Heidelberg, (2012).

[5] C. Bologna, A. C. De Rosa, A. D. Vivo, M. Gaeta, G. Sansonetti and V. Viserta, "Personality-Based Recommendation in E-Commerce", In UMAP Workshops, (2013).

[6] C. Cobos, O. Rodriguez, J. Rivera, J. Betancourt, M. Mendoza, E. LeóN and E. Herrera-Viedma, "A hybrid system of pedagogical pattern recommendations based on singular value decomposition and variable data attributes", Information Processing \& Management 49, no. 3, (2013), pp. 607-625.

[7] F. J. Mata and A. Quesada, "Web 2.0, social networks and e-commerce as marketing tools", Journal of theoretical and applied electronic commerce research, vol. 9, no. 1, (2014), pp. 56-69.

[8] X. Zhao and K. Ji, "Tourism e-commerce recommender system based on web data mining", In Computer Science \& Education (ICCSE), 2013 8th International Conference on, IEEE, (2013), pp. 1485-1488.

[9] R. Tyagi and A. Jawdekar, "An advanced recommendation system for E-commerce users", In Colossal Data Analysis and Networking (CDAN), Symposium on, IEEE, (2016), pp. 1-6.

[10] A. T. Lorente, J. B. Moreno, C. Porcel and E. H. Viedma, "Integrating quality criteria in a fuzzy linguistic recommender system for digital libraries", Procedia Computer Science, vol. 31, (2014), pp. 1036-1043.

[11] C. L. Hsu, J. C. C. Lin and H. S. Chiang, "The effects of blogger recommendations on customers' online shopping intentions", Internet Research, vol. 23, no. 1, (2013), pp. 69-88.

[12] M. Daoud, S. K. Naqvi and A. Ahmad, "Opinion Observer: Recommendation System on ECommerce Website", International Journal of Computer Applications, vol. 105, (2014).

[13] E. B. Kim, "Recommendations for information security awareness training for college students", Information Management \& Computer Security, vol. 22, no. 1, (2014), pp. 115-126.

[14] S. Wei, N. Ye, S. Zhang, X. Huang and J. Zhu, "Collaborative filtering recommendation algorithm based on item clustering and global similarity", In Business Intelligence and Financial Engineering (BIFE), 2012 Fifth International Conference on, IEEE, (2012), pp. 69-72.

[15] A. N. H. Zaied, "Barriers to e-commerce adoption in Egyptian SMEs", International Journal of Information Engineering and Electronic Business, vol. 4, no. 3, (2012), pp. 9.

[16] B. S. Razavi, "Predicting the trend of land use changes using artificial neural network and markov chain model (case study: Kermanshah City)", Research Journal of Environmental and Earth Sciences, vol. 6, no. 4, (2014), pp. 215-226.

[17] D. A. Pomerleau, "Neural network perception for mobile robot guidance", Springer Science \& Business Media, vol. 239, (2012).

[18] C. L. Chen, Y. J. Liu and G. X. Wen, "Fuzzy neural network-based adaptive control for a class of uncertain nonlinear stochastic systems", IEEE Transactions on Cybernetics, vol. 44, no. 5, (2014), pp. 583-593.

[19] A. K. Yadav and S. S. Chandel, "Solar radiation prediction using Artificial Neural Network techniques: A review", Renewable and Sustainable Energy Reviews, vol. 33, (2014), pp. 772-781.

[20] Y. H. Liu, C. L. Liu, J. W. Huang and J. H. Chen, "Neural-network-based maximum power point tracking methods for photovoltaic systems operating under fast changing environments", Solar Energy, vol. 89, (2013), pp. 42-53.

[21] M. H. Esfe, S. Saedodin, M. Bahiraei, D. Toghraie, O. Mahian and S. Wongwises, "Thermal conductivity modeling of $\mathrm{MgO} / \mathrm{EG}$ nanofluids using experimental data and artificial neural network", Journal of Thermal Analysis and Calorimetry, vol. 118, no. 1, (2014), pp. 287-294.

[22] D. Sussillo, M. M. Churchland, M. T. Kaufman and K. V. Shenoy, "A neural network that finds a naturalistic solution for the production of muscle activity", Nature neuroscience, vol. 18, no. 7, (2015), pp 1025-1033.

[23] S. Vaidyanathan, "Anti-synchronization of 3-cells cellular neural network attractors via adaptive control method", International Journal of PharmTech Research, vol. 8, no. 7, (2015), pp. 26-38.

[24] A. Mellit, S. Sağlam and S. A. Kalogirou, "Artificial neural network-based model for estimating the produced power of a photovoltaic module", Renewable energy, vol. 60, (2013), pp. 71-78.

[25] M. Jamshidi, M. Ghaedi, K. Dashtian, A. M. Ghaedi, S. Hajati, A. Goudarzi and E. Alipanahpour, "Highly efficient simultaneous ultrasonic assisted adsorption of brilliant green and eosin B onto ZnS nanoparticles loaded activated carbon: Artificial neural network modeling and central composite design 
optimization”, Spectrochimica Acta Part A: Molecular and Biomolecular Spectroscopy, vol. 153, (2016), pp. 257-267.

[26] L. Zhang, Z. Tao and B. Wang, "SAR image target recognition using kernel sparse representation based on reconstruction coefficient energy maximization rule", In 2016 IEEE International Conference on Acoustics, Speech and Signal Processing (ICASSP), IEEE, (2016), pp. 2369-2373.

[27] H. Wang and J. Wang, "An effective image representation method using kernel classification”, In 2014 IEEE 26th International Conference on Tools with Artificial Intelligence, pp. 853-858. IEEE, (2014).

[28] K. M. Salama, A. M. Abdelbar, F. E. B. Otero and A. A. Freitas, "Utilizing multiple pheromones in an ant-based algorithm for continuous-attribute classification rule discovery", Applied Soft Computing, vol. 13, no. 1, (2013), pp. 667-675.

[29] A. K. Ghosh, P. Chaudhuri and D. Sengupta, "Classification using Kernel density estimates", Technometrics, (2012).

[30] V. V. Ostaptchouk, V. Jana, M. L. Nuotio, S. N. Slagter, D. Doiron, K. Fischer, L. Foco and A. Gaye, "The prevalence of metabolic syndrome and metabolically healthy obesity in Europe: a collaborative analysis of ten large cohort studies", BMC endocrine disorders, vol. 14, no. 1, (2014), pp. 1.

[31] Y. Y. Janjigian, D. Werner, C. Pauligk, K. Steinmetz, D. P. Kelsen, E. Jäger and H. M. Altmannsberger, "Prognosis of metastatic gastric and gastroesophageal junction cancer by HER2 status: a European and USA International collaborative analysis", Annals of oncology, (2012), pp. 104.

[32] E. L. Schymanski, H. P. Singer, J. Slobodnik, I. M. Ipolyi, P. Oswald, M. Krauss and T. Schulze, "Nontarget screening with high-resolution mass spectrometry: critical review using a collaborative trial on water analysis", Analytical and bioanalytical chemistry, vol. 407, no. 21, (2015), pp. 6237-6255.

[33] K. Chan, R. S. Patel, P. Newcombe, C. P. Nelson, A. Qasim, S. E. Epstein and S. Burnett, "Association between the chromosome 9p21 locus and angiographic coronary artery disease burden: a collaborative meta-analysis", Journal of the American College of Cardiology, vol. 61, no. 9, (2013), pp. 957-970.

[34] Y. Huang, X. Wei, T. Wu, R. Chen and A. Guo, "Collaborative care for patients with depression and diabetes mellitus: a systematic review and meta-analysis", BMC psychiatry, vol. 13, no. 1, (2013), pp. 1.

[35] T. Huang, B. Yang, J. Zheng, G. Li, M. L. Wahlqvist and D. Li, "Cardiovascular disease mortality and cancer incidence in vegetarians: a meta-analysis and systematic review", Annals of nutrition and metabolism, vol. 60, no. 4, (2012), pp. 233-240.

[36] N. Wang, Q. Zhang, L. Yang and M. Chen, "A Novel E-commerce Recommendation Algorithm based on Neural Network and Collaborative Analysis", (2016). 